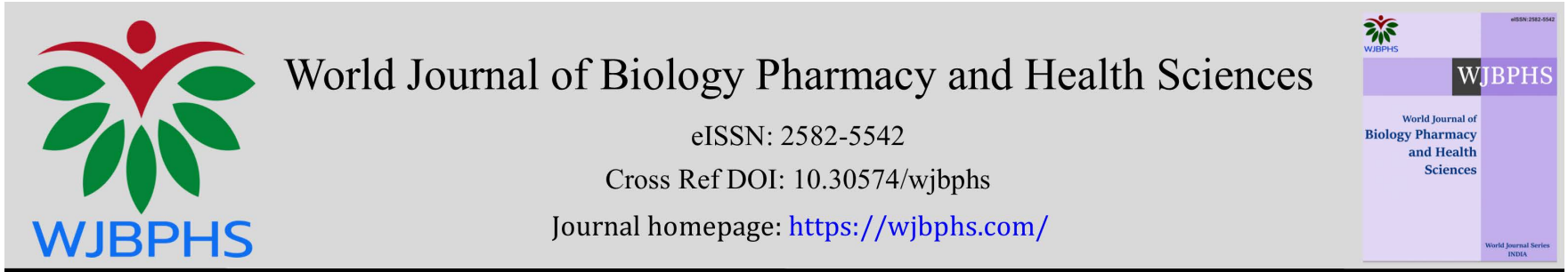

(RESEARCH ARTiCLE)

\title{
Dynamics of organophosphate and carbamate resistance in Anopheles gambiae s. l. populations from south and north Benin, West Africa
}

\author{
Nazaire Aïzoun * \\ Laboratory of Pluridisciplinary Researches of Technical Teaching (LaRPET), Normal High School of Technical Teaching \\ (ENSET) of Lokossa, National University of Sciences, Technologies, Engineery and Mathematics (UNSTIM) of Abomey, P. O. \\ Box 133, Cotonou, Benin.
}

World Journal of Biology Pharmacy and Health Sciences, 2021, 07(01), 023-029

Publication history: Received on 01 May 2021; revised on 12 June 2021; accepted on 15 June 2021

Article DOI: https://doi.org/10.30574/wjbphs.2021.7.1.0066

\begin{abstract}
The current study was aimed to investigate on dynamics of propoxur resistance in Anopheles gambiae s.l. populations from N' dali district in northern Benin (West Africa) and also to investigate on dynamics of malathion resistance in Anopheles gambiae s. l. populations from Toffo district in southern Benin. Larvae and pupae of Anopheles gambiae s. 1 . mosquitoes were collected from the breeding sites in Borgou and Atlantic departments in 2015 and 2019. WHO susceptibility tests were conducted on unfed female mosquitoes aged 2-5 days old. WHO bioassays were performed with impregnated papers with propoxur $0.1 \%$ and with malathion $5 \%$. PCR techniques were used to detect species and Ace-1 mutations in 2015. Anopheles gambiae s. l. populations from N'dali were resistant to propoxur in 2015 and were still remained resistant to this product in 2019. Regarding Anopheles gambiae s. l. populations from Toffo, they were susceptible to malathion in 2015 whereas the malathion resistance status of these mosquitoes requires further investigation in 2019. PCR revealed that all specimens tested were Anopheles gambiae s. s. The presence of Ace-1R at very low frequency (0.01) was observed in Anopheles gambiae s. l. populations from both districts. This study shows that propoxur resistance detected in An. gambiae s. l. populations from N' dali needs to be monitored for insecticide resistance in this area.
\end{abstract}

Keywords: Dynamics; Anopheles gambiae; Resistance; Propoxur; Malathion; Benin

\section{Introduction}

Malaria is a major public health problem in Africa. Achieving universal coverage with effective vector control interventions requires timely and sustained programme-delivery operations. In turn, this requires specialized personal at national, provincial, district and community levels. These teams are best achieved through free mass distribution campaigns every 3 years or less. However, to ensure that coverage is maintained, it is essential to complement these campaigns with continuous distribution programmes (e.g., through antenatal and routine immunization services) before, during and after mass campaigns [1].

In Benin, malaria is one of the most frequently recorded diseases in health centres. Over the past few years, the National Malaria Control Programme (NMCP) has implemented control interventions to reduce the contact between malaria vectors and human hosts. The major control strategies applied at national level were the scaling up of long-lasting insecticidal nets (LLINs) throughout the country and indoor residual spraying (IRS) in one of the cities of Benin (Cotonou). Despite these tremendous efforts made by the NMCP, the results obtained were less encouraging than

\footnotetext{
*Corresponding author: Nazaire Aïzoun

Laboratory ofPluridisciplinary Researches of Technical Teaching (LaRPET), Normal High School of Technical Teaching (ENSET) of Lokossa, National University of Sciences, Technologies, Engineery and Mathematics(UNSTIM) of Abomey, P. 0. Box 133, Cotonou, Benin.
} 
expected. The main problem with ITNs and IRS is the development of insecticide resistance, particularly pyrethroidresistance by several populations of Anopheles gambiae [2-5].

Organophosphates and carbamates (OP and CX) insecticides are competitive inhibitors that irreversibly inhibit the $A C h E$ enzyme, blocking nervous transmission and leading to the death of the insect. So, acetylcholinesterase is a key enzyme in the nervous system, terminating nerve impulses by catalyzing the hydrolysis of the neurotransmitter acetylcholine. It $(A C h E)$ is the major target for organophosphate (OP) and carbamate insecticides, which inhibit enzyme activity by covalently phosphorylating or carbamylating the serine residue within the active site gorge [6].

Resistance monitoring should be seen as a critical element of any medium or large-scale deployment of an insecticidal intervention, and should be overseen and coordinated by National Malaria Control Programmes. This monitoring is usually done by using either World Health Organization (WHO) susceptibility test or the bottle bioassay developed by United States Centers for Diseases Control and Prevention (CDC).

The present study propose was to assess the dynamics of susceptibility to propoxur, a carbamate compound in Anopheles gambiae s. l. populations from northern Benin. To also assess the dynamics of susceptibility to malathion, an organophosphate compound in Anopheles gambiae s. l. populations from south of the country.

\section{Material and methods}

\subsection{Study area}

The study area is located in Republic of Benin (West Africa) and includes the departments of Borgou and Atlantic.

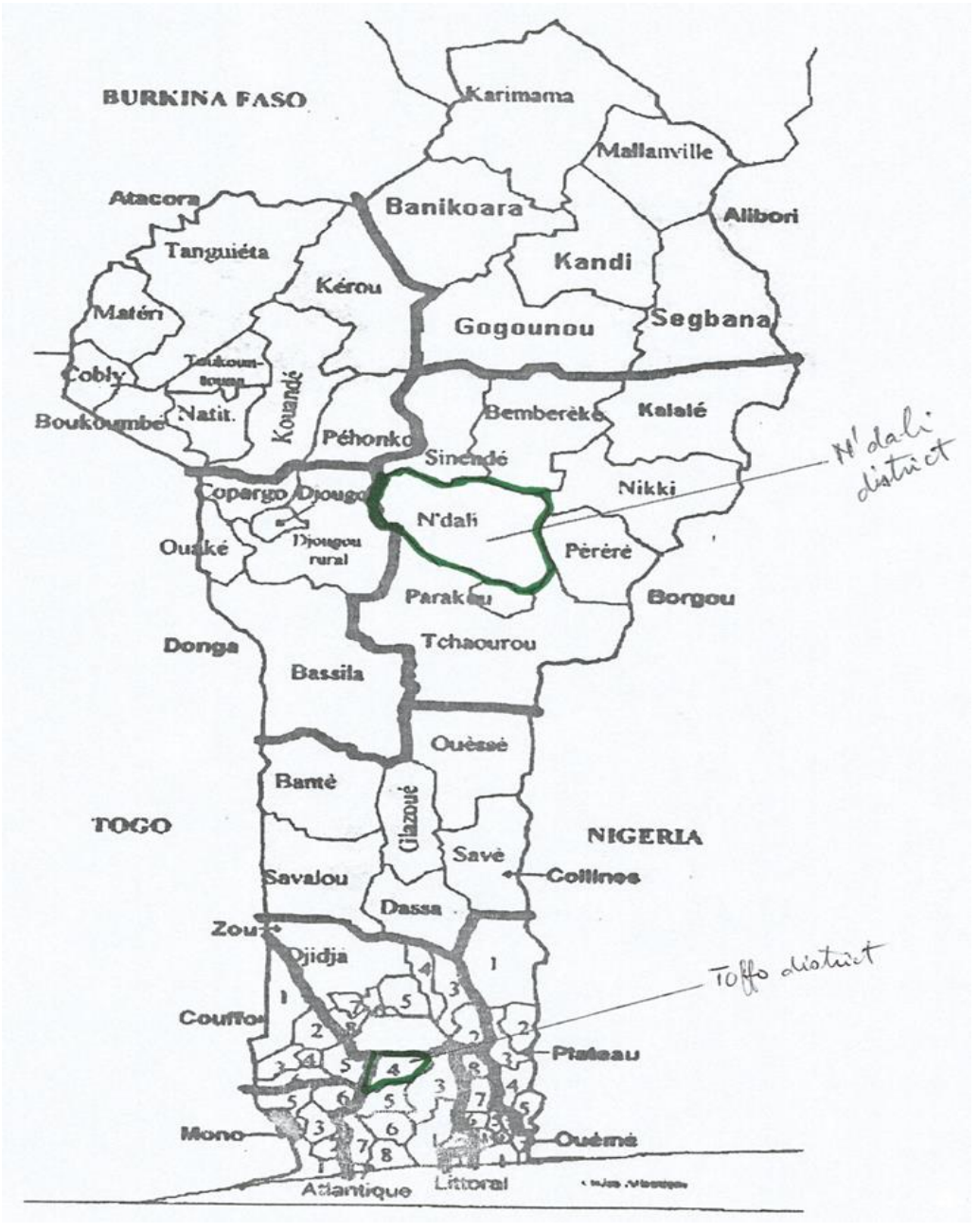

Figure 1 Map of Republic of Benin showing districts surveyed (Source: Council Africa, 2006) 
Borgou department is located in the north of Benin and the study was carried out more precisely in N' dali district, a rice growing area whereas the Atlantic department is located in the south of Benin and the study was carried out more precisely in Toffo district, cereals and fruit growing area. The choice of the study site took into account the economic activities of populations, their usual protection practices against mosquito bites, and peasant practices to control farming pests. The northern zone is characterized by a Sudanian climate with only one rainy season per year (May to October) and one dry season (November to April). The temperature ranged from 22 to $33^{\circ} \mathrm{C}$ with the annual mean rainfall of $1,300 \mathrm{~mm}$ whereas Toffo is characterized by a subequatorial type climate with two rainy seasons (March to July and September to November) in southern Benin. The mean annual rainfall is over 1,500 $\mathrm{mm}$.

\subsection{Mosquito sampling}

An. gambiae s. l. mosquitoes were collected from May to October in 2015 and 2019 during the rainy season in N'dali district selected in the northern part of the country. Anopheles gambiae s. l. mosquitoes were also collected during the rainy seasons (March to July and September to November in 2015 and 2019) across Toffo district selected in southern Benin. Anopheles preimaginal stages (L1 to L4 instars) were collected via ladles within rice farms from N'dali district using the dipping method on several breeding sites and via both padding and town within the cereals and fruit growing area of Toffo district using the dipping method on several breeding sites (brick pits, pools, marshes, streams, ditches, pits dug for plastering traditional huts, puddles of water, water pockets caused by the gutters).

Once, larvae and pupae collected, they were then kept in labeled bottles related to both districts surveyed. Otherwise, larvae collected from multiple breeding sites were pooled together then re-distributed evenly in development trays containing tap water. Larvae were provided access to powdered TetraFin $®$ fish food, and were reared to adults under insectary conditions of $25+/-2^{\circ} \mathrm{C}$ and 70 to $80 \%$ relative humidity at Center of Entomological Researches of Cotonou (CREC) located in Akpakpa, in Cotonou district in 2015 and in insectary of the Laboratory of Applied Entomology and Vector Control (LAEVC) of the Department of Sciences and Agricultural Techniques located in Dogbo district in southwestern Benin in 2019. An. gambiae Kisumu, a reference susceptible strain was used as a control for the bioassay tests. Susceptibility tests were done following WHO protocol on unfed females' mosquitoes aged 2-5 days old reared from larval and pupal collections. All susceptibility tests were conducted in 2015 in the CREC laboratory at $25+/-2^{\circ} \mathrm{C}$ and 70 to $80 \%$ relative humidity whereas all WHO susceptibility tests were conducted in 2019 in the LAEVC.

\subsection{Testing insecticide susceptibility}

The principle of the WHO bioassay is to expose insects to a given dose of insecticide for a given time to assess susceptibility or resistance. The standard WHO discriminating dosages are twice the experimentally derived $100 \%$ lethal concentration (LC100 value) of a reference susceptible strain [7]. In this study, the insecticide tested was propoxur $0.1 \%$ and malathion $5 \%$. The choice of propoxur was justified by bendicarb, an insecticide of the same compound used for Indoor Residual Spraying (IRS) in 2011 in Atacora department which department has a boundary with Borgou department. The choice of malathion was justified by an insecticide of same class as pyrimiphos-methyl which was used for IRS in Atacora in 2013.

An aspirator was used to introduce 20 to 25 unfed female mosquitoes aged 2-5 days into five WHO holding tubes (four tests and one control) that contained untreated papers. They were then gently blown into the exposure tubes containing the insecticide impregnated papers. After one-hour exposure, mosquitoes were transferred back into holding tubes and provided with cotton wool moistened with a $10 \%$ honey solution. The number of mosquitoes "knocked down" at 60 minutes and mortalities at 24 hours were recorded following the WHO protocol [7].

\subsection{PCR detection of species and Ace-1 mutation}

Specimens of An. gambiae populations from the WHO bioassay tests in 2015 were subjected to the An. gambiae species specific PCR assays for species identification [8]. The PCR-Restricted Fragment Length Polymorphism (PCR-RFLP) diagnostic test was used to detect the presence of G119S mutation (ace.1R gene) as described by Weill et al. [9]. Mosquito genomic DNA was amplified using the primers Ex3AGdir 5' GATCGTGGACACCG TGTTCG3' and Ex3AGrev 5'AGGAT GGCCCGCT GGAA CAG3' according to Weill et al. [9]. One microlitre of total DNA extracted from a single mosquito was used as a template in a $25 \mu \mathrm{l}$ PCR reaction containing Taq DNA polymerase buffer, $0.2 \mathrm{mM}$ dNTP and 10 pmol of each primer. The PCR conditions were $94^{\circ} \mathrm{C}$ for $5 \mathrm{~min}$ and then 35 cycles of $\left(94^{\circ} \mathrm{C}\right.$ for $30 \mathrm{~s}, 54^{\circ} \mathrm{C}$ for $30 \mathrm{~s}$ and $72^{\circ} \mathrm{C}$ for $30 \mathrm{~s}$ ) with a final 5 min extension at $72^{\circ} \mathrm{C}$. Fifteen microlitres of PCR product were digested with $5 \mathrm{U}$ of AluI restriction enzyme (Promega) in a final volume of $25 \mu$ l. The PCR fragments were fractionated on a $2 \%$ agarose gel stained with ethidium bromide and visualized under UV light. 


\subsection{Statistical analysis and data interpretation}

The resistance status of mosquito samples was determined according to the WHO criteria [10] as follows:

- Mortality rates between $98 \%-100 \%$ indicate full susceptibility

- Mortality rates between $90 \%-97 \%$ require further investigation

- Mortality rates $<90 \%$, the population is considered resistant to the tested insecticides.

Abbott s formula was not used in this study for the correction of mortality rates in test tubes because the mortality rates in all control tubes was less than $5 \%$ [11].

To compare the status of insecticide resistance, Fisher's exact test was carried out to determine if there was any significant difference between mortality rates of populations of An. gambiae s. s. of district using Statistica 6.0.

Allelic frequency of G119S mutation was analysed using the version 1.2 of Genepop [12]. The software R-2.15.2 (R Development Core Team, 2011) was used for the statistical analysis.

\section{Results}

\subsection{Evolution of Anopheles gambiae s. l. resistance to propoxur in N' dali district from 2015 to 2019}

The Kisumu strain (control) confirmed its susceptibility status as a reference strain. All female mosquitoes of Anopheles gambiae s. $l$. Kisumu that were exposed to WHO impregnated papers with propoxur $0.1 \%$ were dead and none of them could fly after $24 \mathrm{~h}$ mortality recording required by WHO. These results confirmed that this strain was fully susceptible to this product (Table 1 ).

Regarding field collected Anopheles gambiae s. l. population, female mosquitoes of Anopheles gambiae s. l. from N' dali that were exposed to WHO impregnated papers with propoxur $0.1 \%$ were resistant to this product in 2015 and still remained resistant to this product in 2019 with mortalities rate of $83 \%$ and $77 \%$ respectively (Table 1 ).

Table 1 Mortality of An. gambiae s. l. populations from N'dali district after one-hour exposure to WHO impregnated papers with propoxur $0.1 \%$ in 2015 and 2019

\begin{tabular}{|c|c|c|c|c|c|}
\hline Locations & Years & Insecticide & Number tested & \% Mortality & Resistance status \\
\hline \multirow{2}{*}{ Kisumu (Control) } & 2015 & Propoxur & 100 & 100 & $\mathrm{~S}$ \\
\cline { 2 - 6 } & 2019 & Propoxur & 100 & 100 & $\mathrm{~S}$ \\
\hline \multirow{2}{*}{ N'dali } & 2015 & Propoxur & 100 & 83 & $\mathrm{R}$ \\
\cline { 2 - 6 } & 2019 & Propoxur & 100 & 77 & $\mathrm{R}$ \\
\hline
\end{tabular}

\subsection{Evolution of Anopheles gambiae s. l. resistance to malathion in Toffo district from 2015 to 2019}

The Kisumu strain (control) confirmed its susceptibility status as a reference strain. All female mosquitoes of Anopheles gambiae s. $l$. Kisumu that were exposed to WHO impregnated papers with malathion 5\% were dead and none of them could fly after $24 \mathrm{~h}$ mortality recording required by WHO. These results confirmed that this strain was fully susceptible to this product (Table 2).

Regarding field collected female Anopheles gambiae s. l. populations from Toffo that were exposed to WHO papers impregnated with malathion 5\% were susceptible to this product in 2015 with the mortality rate of $99 \%$ whereas the malathion resistance status of these mosquitoes requires further investigation in 2019 (Table 2). 
Table 2 Mortality of An. gambiae s. l. populations from Toffo district after one-hour exposure to WHO impregnated papers with malathion $5 \%$ in 2015 and 2019

\begin{tabular}{|c|c|c|c|c|c|}
\hline Locations & Years & Insecticide & Number tested & \% Mortality & Resistance status \\
\hline \multirow{2}{*}{ Kisumu (Control) } & 2015 & Malathion & 100 & 100 & $\mathrm{~S}$ \\
\cline { 2 - 6 } & 2019 & Malathion & 100 & 100 & $\mathrm{~S}$ \\
\hline \multirow{2}{*}{ Toffo } & 2015 & Malathion & 100 & 99 & $\mathrm{~S}$ \\
\cline { 2 - 6 } & 2019 & Malathion & 100 & 93 & $\mathrm{r}$ \\
\hline
\end{tabular}

\subsection{Mosquito species identification}

PCR revealed that $100 \%$ of mosquitoes tested in N'dali district and in Toffo district were Anopheles gambiae s. s. (Tables 3 and Table 4).

\subsection{Detection of Ace-1 mutation}

The results of molecular tests performed on Anopheles gambiae populations from N'dalii district (Table 3) and from Toffo district (Table 4) revealed that the frequencies of Ace-1 mutation were $1 \%$.

Table 3 Ace-1 mutation frequency in An. gambiae populations from N'dali district in 2015

\begin{tabular}{|l|c|l|l|l|l|c|}
\hline & & & \multicolumn{4}{|c|}{ Ace-1 mutation } \\
\hline Location & Number tested & Species Ag & RR & RS & SS & F(Ace-1) \\
\hline N'dali & 47 & 47 & 0 & 1 & 46 & 0.01 \\
\hline
\end{tabular}

Table 4 Ace-1 mutation frequency in An. gambiae populations from Toffo district in 2015

\begin{tabular}{|l|l|l|l|l|l|l|}
\hline \multicolumn{2}{|l|}{} & \multicolumn{4}{l|}{ Ace-1mutation } \\
\hline Location & Number tested & Species Ag & RR & RS & SS & F(Ace-1) \\
\hline Toffo & 55 & 55 & 0 & 1 & 54 & 0.01 \\
\hline
\end{tabular}

Ag: An. Gambiae s. S.; RR: Homozygous Resistant; RS: Heterozygous; SS: Homozygous Susceptible; F (Ace-1): Frequency of Ace-1

\section{Discussion}

Anopheles gambiae, which is the main malaria vector in Benin has developed high level of resistance to pyrethroid insecticides. This raises serious concerns to the future use of long-lasting insecticidal nets (LLIN) and indoor residual spraying (IRS). It is therefore important to seek better and effective resistance management strategies which will use organophosphates or carbamates as alternatives against pyrethroid resistant malaria vectors in the field.

Female Anopheles gambiae s. l. population from N' dali which were exposed to WHO impregnated papers with propoxur $0.1 \%$ were resistant to this product in 2015 and still remained resistant to this product in 2019. So, An. gambiae s. . populations from N'dali were resistant to propoxur in northern part of the country. According to N' Guessan et al. [13], An. gambiae breeds in rice fields (at early stage of the cultivation) and habitually rests in houses of urban and peri-urban areas, and it may be exposed to considerable selection pressure by agricultural insecticides and/or domestic aerosols and mosquito coils. Carbamate resistance was also recently reported in An. gambiae populations from Atacora department in north-western Benin $[14,15]$ with the implication of biochemical resistance mechanisms mainly the esterases activity in bendiocarb resistance [16]. In addition, Djogbenou et al. (2008, unpublished data) has shown that Anopheles gambiae Kandi populations from Alibori department in northern Benin were also resistant to carbosulfan, a carbamate, with a mortality rate of $73 \%$.

Female Anopheles gambiae s. l. populations from Toffo were susceptible to malathion. This result may be explained by the low amount of insecticidal products (OP) applied in agriculture and public health in this district. The susceptibility 
to malathion in An. gambiae s.l. populations from southern Benin was already reported by Corbel et al. [17] more precisely in Ladji and Asecna localities in Cotonou district. This study by Corbel et al. [17] suggested that there were no malathion-specific carboxyesterases in Benin.

Propoxur resistance in An. gambiae N' dali populations was corroborated with Ace-1 mutation frequency as it was $1 \%$. This result confirmed that there was really resistance of these populations to this carbamate compound.

In the current study, PCR revealed that $100 \%$ of mosquitoes from N' dali tested were Anopheles gambiae s. s. No An. arabiensis mosquitoes were found. Conversely, Djogbenou et al. (2008, unpublished data) has shown that Anopheles arabiensis populations were also present in N' dali in the proportion of $1.2 \%$ within An. gambiae complex. So, this result showed that An. arabiensis populations from $\mathrm{N}$ dali district tend to decline.

\section{Conclusion}

Carbamate resistance detected in Anopheles gambiae, vector of malaria, in Atacora department in north-western Benin is spreadto surrounding departments of Atacora department in northern Benin like Borgou department. Even if organophosphate resistance is already present in Anopheles gambiae s. l. populations from northern Benin, those from southern Benin are still susceptible to this compound. Further studies are needed to investigate the use of malathion for Indoor Residual Spraying in order to guide future malaria vector control interventions by National Malaria Control Programme (NMCP) in Benin.

\section{Compliance with ethical standards}

\section{Acknowledgments}

I would like to thank people from localities surveyed who had helped us in mosquito collection. I would also like to thank KOUASSI Prisca for technical assistance in laboratory during the current study.

\section{Disclosure of conflict of interest}

I declare there is no conflict of interest.

\section{References}

[1] WHO. World Malaria Report. Geneva, World Health Organization. 2013.

[2] Elissa N, Mouchet J, Rivière F, Meunier JY, Yao K. Resistance of Anopheles gambiae s. s. to pyrethroids in Côte d'Ivoire . Ann Soc Belg Med Trop. 1993; 73: 291-294.

[3] Akogbéto M, Yakoubou S. Resistance of malaria vectors to pyrethroids used for mosquito nets treated in Benin, West Africa . Bull Soc Pathol Exot. 1999; 92: 123-130.

[4] Chandre F, Darriet F, Duchon S, Finot L, Manguin S, Carnevale P, et al. Modifications of pyrethroid effects associated win kdr mutation in Anopheles gambiae. Med Vet Entomol. 2000; 14: 81-88.

[5] Akogbeto M, Djouaka RF, Kinde-Gazard DA. Screening of pesticide residues in soil water samples from agricultural settings. Malar J. 2006; 5: 22.

[6] Corbett JR. The Biochemical Mode of Action of Pesticides. Academic Press, New York. 1974; 330.

[7] WHO. Report of the WHO Informal Consultation. Tests procedures for insecticide resistance monitoring in malaria vectors, bio-efficacy and persistence of insecticides on treated surfaces. Geneva: World Health Organization: Parasitic Diseases and Vector Control (PVC)/Communicable Disease Control, Prevention and Eradication (CPE). 1998; 43.

[8] Scott JA, Brogdon WG, Collins FH. Identification of single specimens of the Anopheles gambiae complex by the polymerase chain reaction. Am J Trop Med. Hyg. 1993; 49: 520-529.

[9] Weill M, Lutfalla G, Mogensen K, Chandre F, Berthomieu A, Berticat C, et al. Comparative genomics: insecticide resistance in mosquito vectors. Nature (Lond.). 2003; 423: 136-137.

[10] WHO. Test procedures for insecticide resistance monitoring in malaria vector mosquitoes. Geneva: World Health Organization. $2013 \mathrm{~b}$. 
[11] Abbott WS. A method of computing the effectiveness of an insecticide. J Am Mosq Cont Assoc. 1987; 3(2): $302-$ 303.

[12] Raymond M, Rousset F. Genepop (version 1.2), population genetics software for exact tests and eucumenicism. J Heredity. 1995; 86: 248-249.

[13] N'Guessan R, Darriet F, Guillet P, Carnevale P, Traore-Lamizana M, Corbel V, et al. Resistance to carbosulfan in Anopheles gambiae from Ivory Coast, based on reduced sensitivity of acetylcholinesterase. Med Vet Entomol. 2003; 17: 19-25.

[14] Aïkpon R, Agossa F, Ossè R, Oussou O, Aïzoun N, Oké-Agbo F, et al. Bendiocarb resistance in Anopheles gambiae s. l. populations from Atacora department in Benin, West Africa: a threat for malaria vector control. Parasit Vectors. 2013; 6: 192.

[15] Aïzoun N, Aïkpon R, Gnanguenon V, Oussou O, Agossa F, Padonou GG, et al. Status of organophosphate and carbamate resistance in Anopheles gambiae sensu lato from the south and north Benin, West Africa. Parasit Vectors. 2013; 6: 274.

[16] Aïzoun N, Aïkpon R, Padonou GG, Oussou O, Oké-Agbo F, Gnanguenon V, et al. Mixed-function oxidases and esterases associated with permethrin, deltamethrin and bendiocarb resistance in Anopheles gambiae s. l. in the south-north transect Benin, West Africa. Parasit Vectors. 2013; 6: 223.

[17] Corbel V, N Guessan R, Brengues C, Chandre F, Djogbenou L, Martin T, et al. Multiple insecticide resistance mechanisms in Anopheles gambiae and Culex quinquefasciatus from Benin, West Africa. Acta Tropica. 2007; 101: 207-216. 